\title{
Low-resource low-latency hybrid adaptive CORDIC with floating-point precision
}

\author{
Hong-Thu Nguyen ${ }^{1 a)}$, Xuan-Thuan Nguyen ${ }^{1}$, Trong-Thuc Hoang ${ }^{2}$, \\ Duc-Hung Le ${ }^{2}$, and Cong-Kha Pham ${ }^{1}$ \\ ${ }^{1}$ The University of Electro - Communications, \\ 1-5-1 Chofugaoka, Chofu, Tokyo 182-8585, Japan \\ ${ }^{2}$ The University of Science, Ho Chi Minh City, \\ 227 Nguyen Van Cu St., Dist. 5, Vietnam \\ a)hongthu@vlsilab.ee.uec.ac.jp
}

\begin{abstract}
Despite being proposed since more than 50 years ago, COordinate Rotation DIgital Computer (CORDIC) is still one of the most effective algorithms for elementary function calculation so far. Original CORDIC, however, suffers high latency due to its nature of unvarying number of rotations. As a result, a low-latency hybrid adaptive (HA) CORDIC is proposed in this paper. Firstly, adaptive angle selection decreases total iterations up to $50 \%$ with respect to higher accuracy of results. Secondly, hybrid architecture including fixed-point input and floating-point output reduces the total hardware utilization and enhances the dynamic range of final results. Lastly, parallel and pipeline processing together with resource sharing technique allow the design to operate fully at $175.7 \mathrm{MHz}$ with low resource consumption - 1,139 LUTs and 489 registers.
\end{abstract}

Keywords: CORDIC, low-latency, low-resource, hybric, floating point Classification: Electron devices, circuits, and systems

\section{References}

[1] J. E. Volder: IRE Trans. Electron. Comput. EC-8 (1959) 330. DOI:10.1109/ TEC. 1959.5222693

[2] J. S. Walther: Proc. Spring Joint Computer Conf. (1971) 379.

[3] P. K. Meher, J. Valls, T.-B. Juang, K. Sridharan and K. Maharatna: IEEE Trans. Circuits Syst. I, Reg. Papers 56 (2009) 1893. DOI:10.1109/TCSI.2009. 2025803

[4] A. M. Despain: IEEE Trans. Comput. C-23 (1974) 993. DOI:10.1109/T-C. 1974.223800

[5] E. H. Wold and A. M. Despain: IEEE Trans. Comput. C-33 (1984) 414. DOI:10.1109/TC.1984.1676458

[6] R. Touzi, R. K. Hawkins and S. Cote: IEEE Trans. Geosci. Remote Sens. 51 (2013) 487. DOI:10.1109/TGRS.2012.2201946

[7] D. M. Munoz, D. F. Sanchez, C. H. Llanos and M. Ayala-Rincón: SPL VI Southern Programmable Logic Conf. (2010) 55. DOI:10.1109/SPL.2010. 5483002

[8] P. Surapong and M. Glesner: FPL Int. Conf. Field Programmable Logic and Applications (2011) 382. DOI:10.1109/FPL.2011.74 
[9] N. Dhume and R. Srinivasakannan: Xilinx Application Note XAPP552 (2012) 1.

[10] J. Zhou, Y. Dong, Y. Dou and Y. Lei: ICESS Int. Conf. Embedded Software and Systems (2008) 616. DOI:10.1109/ICESS.2008.95

[11] Y. H. Hu and S. Naganathan: IEEE Trans. Comput. 42 (1993) 99. DOI:10. $1109 / 12.192217$

[12] T. K. Rodrigues and E. E. Swartzlander: IEEE Trans. Comput. 59 (2010) 522. DOI:10.1109/TC.2009.190

[13] P. K. Meher and S. Y. Park: IEEE Trans. Very Large Scale Integr. (VLSI) Syst. 21 (2013) 217. DOI:10.1109/TVLSI.2012.2187080

[14] R. Shukla and K. C. Ray: IEEE Trans. Comput. 63 (2014) 3066. DOI:10.1109/ TC. 2013.173

[15] S. Aggarwal, P. K. Meher and K. Khare: IEEE Trans. Circuits Syst. I, Reg. Papers 60 (2013) 314. DOI:10.1109/TCSI.2012.2215778

[16] Y. Lei, Y. Dou, S. Guo and J. Zhou: LNCS Adv. Parallel Process. Technol. 6965 (2011) 127. DOI:10.1007/978-3-642-24151-2_10

[17] Altera: Floating-Point Megafunctions User Guide (2013) 1.

\section{Introduction}

CORDIC, a simple and efficient iterative algorithm to compute elementary functions, was first introduced by Volder [1] in 1959 and later extended by Walther [2] in 1971. In fact, CORDIC only requires the additions, subtractions, and shift operations, thereby fitting with Very-Large-Scale Integration (VLSI) system design. For this reason, a vast amount of research in CORDIC algorithm and hardware solution still are in progress although CORDIC is more than 50 years old [3].

CORDIC-based Fast Fourier Transform (FFT) $[4,5]$ plays an essential role in most multimedia and wireless communication applications, where the evaluation of trigonometric functions are imperative. Fixed-point number representation is widely utilized in those systems due to its simple calculation and sufficient precision. However, some advanced applications such as Synthetic Aperture Radar (SAR) data processing [6], require not only highly precise results but also suitable format to manage wide dynamic range of numbers. In those systems, floating-point representation, instead of fixed-point, are deployed to retain real numbers resolution and accuracy effectively. Many approaches to highly efficient floating-point CORDIC, therefore, have been proposed recently. D. M. Munoz et al. [7] described a two-operation-mode floating-point CORDIC architecture that can compute the sine, cosine, or arctangent function. The design achieved an operating frequency of 86.1 MHz with elapsed time around 90 clock cycles at single-precision configuration. P. Surapong et al. [8] proposed an 8- and 16-stage pipelined floating-point CORDIC for phase and magnitude detector. As much as, 23\% slice register and $38 \%$ slice lookup table (LUT) of Xilinx Virtex-5 are used for this 16-stage system, whereas the maximum frequency is only 133.8 MHz. Both Nikhil Dhume et al. [9] and Jie Zhou et al. [10] presented a hybrid approach that firstly convert floatingpoint input into fixed-point format. A fixed-point CORDIC, then, computes the trigonometric functions and those results, lastly, are transformed into IEEE 754 floating-point format. 
In order to reduce error in the results of CORDIC system, more iterations must be performed. However, the number of iterations and clock cycles of each iteration significant affect the latency of CORDIC algorithm. Many approaches to enhance the precision without sacrificing the latency, therefore, have been increasingly attractive. In 1993, Y. H. Hu et al. [11] proposed a method called angle recording which could reduce $50 \%$ the number of iterations. K. R. Terence et al. [12] proposed parallel angle recording method that converged to the final result in the least number of iterations. This method chooses all angle constants in one step but requires a large number of comparison logics. P. K. Meher et al. [13] used angle recording scheme for rotating a fixed angle that could be used in specific application areas such as robotics, graphics, games, and animation. R. Shukla et al. [14] proposed a new low-latency CORDIC algorithm that combining two existing algorithms. This new one can reduce the iterations to $(3 n / 8+1)$ with $n$ is the number of bit precision. Likewise, another reducing iteration algorithm was proposed by S. Aggarwal et al. [15], which could be applied to waveform generator.

In this paper, a low-latency hybrid adaptive CORDIC with floating-point precision is proposed. Moreover, this proposed scheme can obtain low-resource consumption. The contributions of this research are described in detail as follows.

- Low-resource: Hybrid architecture includes 24-bit fixed-point input angle in degree and 32-bit IEEE 754 floating-point sine/cosine outputs. This architecture aims to balance the calculation accuracy with resource utilization. Furthermore, in floating-point arithmetic, resource sharing technique is implemented to reduce the logic utilization by around $70 \%$ in comparison with Altera library of the same function.

- Low-latency: Parallel processing is applied to fixed- and floating-point components, and pipeline processing is deployed in each of them to improve the throughput as well as latency. Moreover, adaptive technique, which reduces the number of iterations by obtaining the final results in the least number of constant angles, is an important part to reduce the latency.

- High-precision: Adaptive technique and hybrid architecture improve not only latency and resource consumption, respectively, but also the precision of the proposed system.

\section{Proposed CORDIC algorithm}

\subsection{Overview of conventional CORDIC algorithm}

CORDIC algorithm consists of two operation modes namely vectoring and rotation mode. In this paper, rotation mode is focused on to calculate sine and cosine of an input angle $\Phi$. Initially, the initial vector of CORDIC algorithm is $V_{0}\left(x_{0}, y_{0}\right)$. After each micro-rotation $i$, this vector is adjusted by an angle constant $\theta_{i}$ as long as residual angle $z_{i}$ approaches to zero. The equation to calculate $x_{i+1}, y_{i+1}$ which do not contain gain factor $k_{i}$ in each iteration of rotation mode is (1). 


$$
\begin{aligned}
& x_{i+1}=x_{i}-d_{i} y_{i} 2^{-i} \\
& y_{i+1}=y_{i}+d_{i} x_{i} 2^{-i} \\
& z_{i+1}=z_{i}-d_{i} \theta_{i} \\
& d_{i}=\operatorname{sign}\left(z_{i}\right)
\end{aligned}
$$

Gain factor, $K$ is used to eliminate $k_{i}$ multiplication at each iteration $i$, where $N$ is the total predetermined angles.

$$
K=\prod_{i=0}^{N-1} k_{i}=\prod_{i=0}^{N-1} \cos \theta_{i}
$$

Trigonometric results, finally, are derived from $x_{N-1}, y_{N-1}$ and $K$ through (3), and sum of $\theta_{i}$ almost reaches input angle $\Phi$.

$$
\begin{aligned}
& \sin (\Phi)=x_{N-1} * K \\
& \cos (\Phi)=y_{N-1} * K \\
& \Phi=\sum_{i=0}^{N-1} d_{i} \theta_{i}
\end{aligned}
$$

\subsection{Proposed hybrid adaptive (HA) CORDIC algorithm}

The HA-CORDIC algorithm is proposed to reduce the number of iterations and thereby reduces the latency. In other words, only several angles in the set of $N$ angle constants are utilized to form the closest result. This proposed optimized 4-step algorithm developed for sine/cosine calculation is shown in Fig. 1. To begin with, the input angle is converted into predefined range. Secondly, an optimum set of micro-rotations whose sum approximates the input angle are selected. The coordinate $(x, y)$, and $K$ factor, then, are correspondingly updated. Finally, sine and cosine values are achieved by the product of latest $x_{t}, y_{t}$, and $K$, together with simple adjustments.

\subsubsection{Angle normalization}

The CORDIC algorithm will only converge across a limited range of input values. In rotation mode, convergence is guaranteed for the angles below the sum of entire $N$ angle constants, i.e. between $-99.88^{\circ}$ and $99.88^{\circ}$. However, all of the trigonometric values of any angle in circular can be interpolated from trigonometric value of an angle in range $\left[0^{\circ}, 45^{\circ}\right]$. Therefore, a trigonometric identity is used to translate any outside-range angle into that within this range. By employing several simple adjustments, outside-range angles can be shortened the range $\left[0^{\circ}, 45^{\circ}\right]$.

\subsubsection{Angle selection}

Assume that 16 angle constants, $\theta$, are employed in the proposed CORDIC as shown in Table I. Conventional CORDIC exploits all $\theta_{i}$ angles and their direction $d_{i}$ for trigonometric computations, as determined in (1). For example, $30^{\circ}$ input angle costs 16 iterations. 


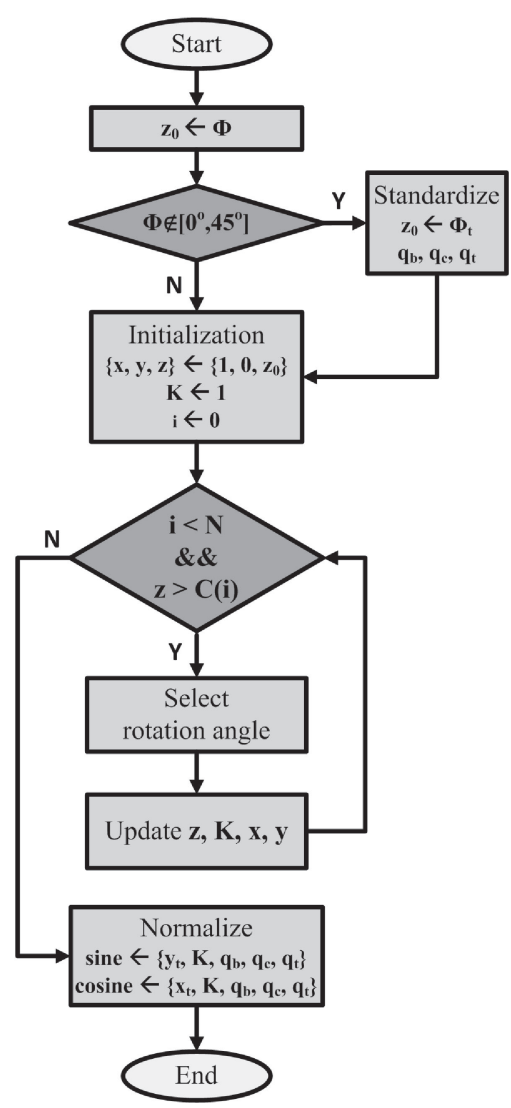

Fig. 1. The flow chart of HA-CORDIC algorithm.

$$
\begin{aligned}
& \left(\theta_{0}-\theta_{1}+\theta_{2}-\theta_{3}+\theta_{4}+\theta_{5}-\theta_{6}+\theta_{7}-\theta_{8}\right. \\
& \left.\quad-\theta_{9}+\theta_{10}+\theta_{11}-\theta_{12}+\theta_{13}-\theta_{14}-\theta_{15}\right)=30.000834^{\circ}
\end{aligned}
$$

In case the input angle is $30^{\circ}$, the residual angle of conventional CORDIC is $8.34 e-4$. The proposed method, however, only requires five iterations to achieve better residual angle, $2.45 e-4$.

$$
\left(\theta_{1}+\theta_{4}-\theta_{9}-\theta_{11}-\theta_{15}\right)=29.999755^{\circ}
$$

The key point of this method is that in each iteration $i$, angle constant $\theta_{i}$ is selected so that residual angle $z_{i}$ converges to zero. The iteration stops upon $z_{i}$ is smaller than a predefined threshold.

In order to choose the angle constant in each iteration, this method uses a set of parameters, $C$, which expresses the range of residual angles around one angle constant, as defined in (5). The details of $C$ is described in Table I. On this basis, the pseudocode determines which angle constant is chosen summarized in Fig. 2.

$$
c_{i}= \begin{cases}\frac{\theta_{i}+\theta_{i+1}}{2} & \text { if } 0 \leq i \leq(N-2) \\ \frac{\theta_{i}}{2} & \text { otherwise }\end{cases}
$$


Table I. The values of $\theta, C$, and $K$.

\begin{tabular}{c|c|c|c}
\hline $\mathbf{i}$ & $\theta$ & $\mathbf{C}$ & $\mathbf{K}$ \\
\hline $\mathbf{0}$ & 45.000000000 & 35.782525588 & 0.707106781 \\
\hline $\mathbf{1}$ & 26.565051177 & 20.300647322 & 0.894427191 \\
\hline $\mathbf{2}$ & 14.036243468 & 10.580629908 & 0.970142500 \\
\hline $\mathbf{3}$ & 7.125016349 & 5.350675362 & 0.992277877 \\
\hline $\mathbf{4}$ & 3.576334375 & 2.683122491 & 0.998052578 \\
\hline $\mathbf{5}$ & 1.789910608 & 1.342542159 & 0.999512076 \\
\hline $\mathbf{6}$ & 0.895173710 & 0.671393940 & 0.999877952 \\
\hline $\mathbf{7}$ & 0.447614171 & 0.335712335 & 0.999969484 \\
\hline $\mathbf{8}$ & 0.223810500 & 0.167858088 & 0.999992371 \\
\hline $\mathbf{9}$ & 0.111905677 & 0.083929284 & 0.999998093 \\
\hline $\mathbf{1 0}$ & 0.055952892 & 0.041964672 & 0.999999523 \\
\hline $\mathbf{1 1}$ & 0.027976453 & 0.020982340 & 0.999999881 \\
\hline $\mathbf{1 2}$ & 0.013988227 & 0.010491170 & 0.999999970 \\
\hline $\mathbf{1 3}$ & 0.006994114 & 0.005245585 & 0.999999993 \\
\hline $\mathbf{1 4}$ & 0.003497057 & 0.002622792 & 0.999999998 \\
\hline $\mathbf{1 5}$ & 0.001748528 & 0.000874264 & 0.999999999 \\
\hline & & &
\end{tabular}

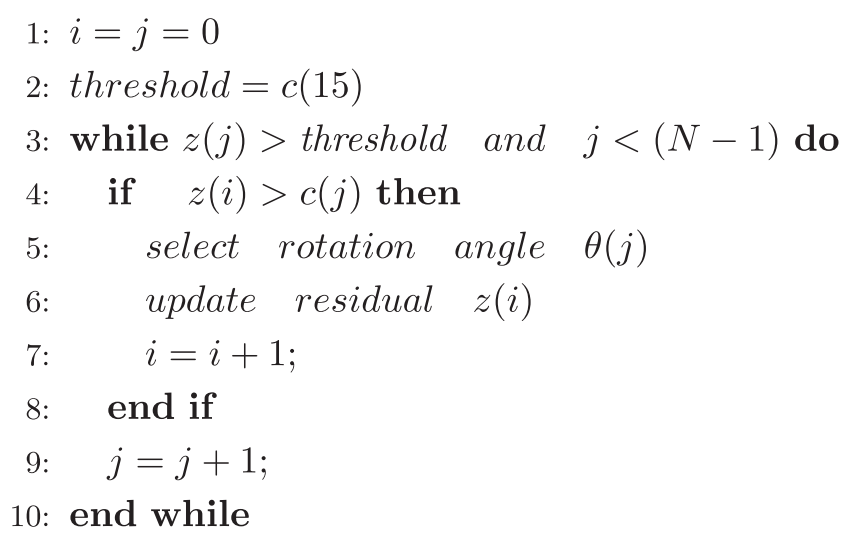

Fig. 2. The pseudocode of angle selection function.

\subsubsection{Pre-calculation: coordinate, residual, and factor adaption}

The adaption of coordinate $(x, y)$, residual $z$, and factor $K$ are described in (6). Because only several angles are selected, factor $2^{-i}, \theta_{i}$, and $k_{i}$ are replaced by $2^{-j}$, $\theta_{j}$, and $k_{j}$ respectively. Both $j$ and $\theta_{j}$ are obtained in Fig. 2, where $k_{j}$ is listed in Table I.

$$
\begin{aligned}
x_{i+1} & =x_{i}-d_{i} y_{i} 2^{-j} \\
y_{i+1} & =y_{i}+d_{i} x_{i} 2^{-j} \\
z_{i+1} & =z_{i}-d_{i} \theta_{j} \\
K & =K * k_{j}
\end{aligned}
$$

If the residual is smaller than the defined threshold, the latest coordinate $\left(x_{t}, y_{t}\right)$, and gain factor $K$ can be achieved. Finally, $X, Y$ value can be obtained by (7). 


$$
\begin{aligned}
& X=x_{t} * K \\
& Y=y_{t} * K
\end{aligned}
$$

\subsubsection{Post-calculation: sine/cosine recovery}

Before coordinate $(X, Y)$ is calculated, the input angle is normalized, thereby the final sine and cosine results of the input angle must be recovered from $(X, Y)$ values.

\section{Proposed hardware architecture}

\subsection{Overview}

The proposed design is composed of four main modules namely ANGLE_SELECTION (ASEL), FIFO, PRE_CALCULATION (PREC), and POST_CALCULATION (POSC), which are illustrated in Fig. 3a. The input is 24-bit fixed-point (FIX) angle that format is 1.8.15, i.e. 1-bit sign, 8-bit magnitude, and 15-LSBs, and the outputs are two 32-bit floating-point (FLP) trigonometric results.

The processing of HA-CORDIC is separated into two parallel threads, which can be seen from Fig. 3b. Because of the difference in operating cycles, a FIFO is inserted between ASEL and PREC to ease the latency. In fact, ASEL and PREC/ POSC cost one and two clock cycles for FIX and FLP operation, respectively. Besides, pipeline processing is applied to all modules to increase the throughput. Depending on the number of iterations is determined in ASEL, the execution latency of each angle is markedly different. Each module is described in more detail below.

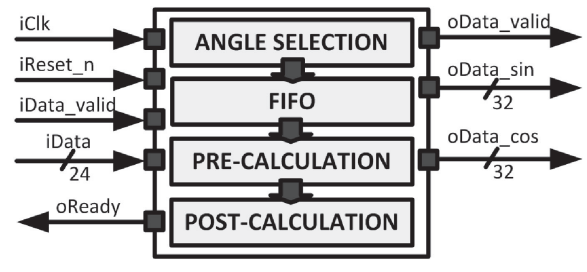

(a)

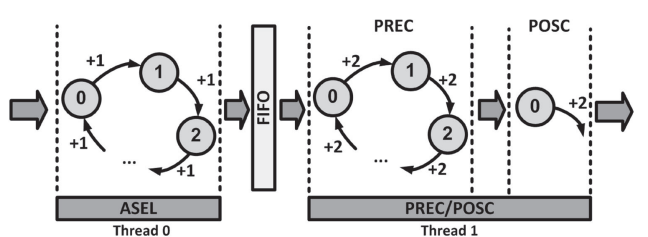

(b)

Fig. 3. (a) The general block diagram of proposed CORDIC. (b) The mechanism of proposed CORDIC.

\subsection{Angle selection (ASEL)}

Module ASEL strives to obtain the precise result with the least number of iterations by reducing divergent pseudo-rotations. This module is composed of three main components, ANGLE_NORMALIZER (ANOR), SET_NEXT_ROTATION (SNR), and CHECK_LAST_ROTATION (CLR), which are depicted in Fig. 4. Beforehand, ANOR converts input angle iData into normalization range of $\left[0^{\circ}, 45^{\circ}\right]$. It can be seen in Fig. 5a, if a circle is split into eight pieces, any angle from the first to the seventh piece can be transformed into equivalent angle in zeroth piece. The recovery information (Rec. info) is transfered to the post-calculation to adjust the final result. By using the Correction equation, the final sine/cosine results can be achieved. 


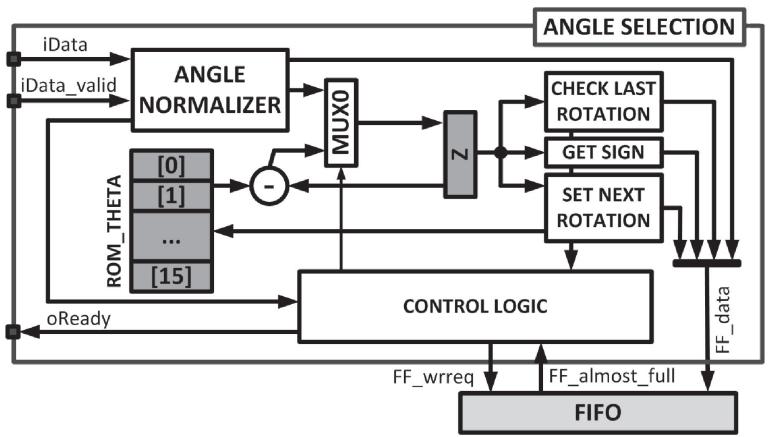

Fig. 4. The hardware architecture of phase search circuit.

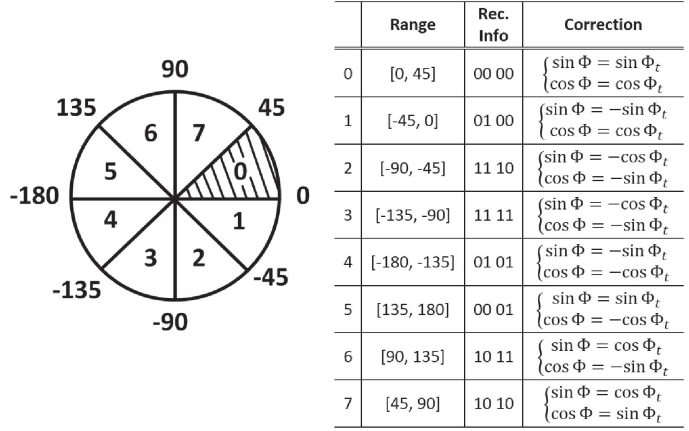

(a)

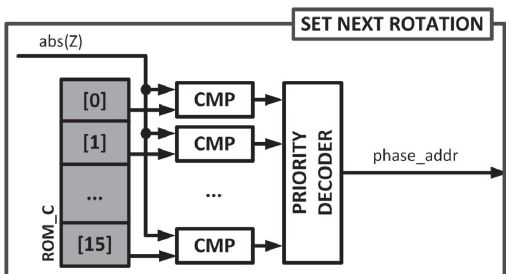

(b)

Fig. 5. (a) A description of normalization technique. (b) The hardware architecture of SET NEXT ROTATION.

After receiving the normalized angle, SNR determines the next angle in ROM_THETA by utilizing a pair of ROM_C and priority encoder, as illustrated in Fig. 5b. Module ROM_THETA includes 16 angle constants $\theta$ while ROM_C stores the range of residual angles $C$ around one angle constant, which is defined in (5). In order to eliminate the while loop in pseudocode from Fig. 2, a set of comparators is deployed in parallel together with a priority encoder to search for next suitable angle $\theta$ at the speed of one cycle. The iteration completes as soon as residual angle register $Z$ is smaller than threshold.

At each iteration $i$, CLR checks whether the current process is the last or not. If the current process is the final iteration, CLR signals ASEL to stop calculating and start the new input angle rotation in following cycle. The pseudocode of this circuit is described in Fig. 6. If input angle $\Phi$ is approximately zero, no rotation is executed (line 5 and 6). If $z$ is within the determined range, updated $z$ will become smaller than the threshold in next rotation (line 7 to 10). Because the addition and subtraction affect the circuit frequency, two LUTs ROM_s $\left(\theta_{i}-\right.$ threshold $)$ and ROM_a $\left(\theta_{i}+\right.$ threshold $)$ are implemented instead. In this hardware system, module SNR and CLR are implemented in parallel.

A collection of 4-bit norm_info, 2-bit lastrotation, 1-bit sign, and 4-bit phase_addr, lastly, is brought together and put into FIFO. At the same time, CONTROL_LOGIC gets FIFO to accept data by asserting FF_wrreq. If FIFO is not available or current angle is still in progress, oReady will go to low level and thereby CORDIC cannot accept new input angle. 


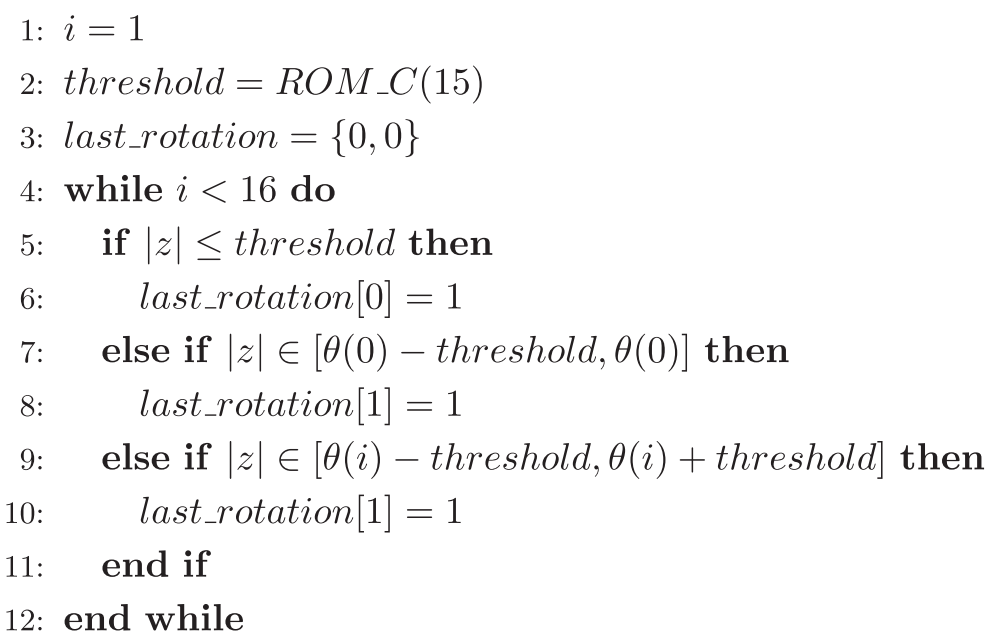

Fig. 6. The pseudocode of angle selection function.

\subsection{Pre-calculation (PREC)}

PREC contains four main components: Floating-point Adder Subtractor (FADD_ SUB), Floating-point Multiplier $k_{i}$ (FMUL_ki), Floating-point Multiplier $X Y K$ (FMUL_XYK), and CONTROL_LOGIC, as shown in Fig. 7a.

FADD_SUB calculates $X$ and $Y$ due to $i$ and $\operatorname{sign} Z$ in each iteration, as illustrated in Fig. 7b. The initial values of $X$ and $Y$ are set as one and zero, respectively, immediately after FADD_SUB is reset. FADD_SUB is active by asserting start within two clock cycles while holding both signZ and $i$. Simultaneously, phase becomes the control signal for multiplexing the data path during the operation. The 4-stage shifter performs a right shift operation with zeros fetched into empty MSB because of the fraction parts of $x$ and $y$. The sign decision checks signZ, phase, and sign of previous $X$ and $Y$ data to decide the operation, addition or subtraction, in Carry Look Ahead (CLA) adder. The two's complement (2's complement), then, will correct the result in case it is a negative number. Finally, all of the information will produce the sign of the result to complete the process of the module.

FMUL_ki produces the gain-factor $K$ by multiplying each step-factor $k_{i}$ in each iteration $i$, as shown in Fig. 7c. Initial value of register RegK in module FMUL_ki is set as one and is sequentially updated by previous $K$ and $\mathrm{ROM}_{-} K$ that is depicted in Table I. FMUL_ki also requires 2-clock-delay start for its pipeline computation.

FMUL_XYK calculates the products of latest $X / Y$ and $K$ whose circuit is illustrated in Fig. 7d. The last signal is set within two clock cycles while remaining the $X$ and $Y$ to enable the FMUL_XYK. The raw cosine and sine results are ready in third and fourth clocks, respectively. The results of sine, cosine pre-calculation are stored in registers regX, regY. This proposed hardware uses parallel and pipeline processing and resource sharing techniques. It can be seen that by using proposed scheme latency, throughput, and hardware utilization are improved significantly.

\subsection{Post-calculation (POSC)}

The raw sine and cosine values are combined with rec_info to form the final trigonometric results. The recovery information rec_info given in Fig. 5a is utilized to select the suitable adjusted pre_sin or pre_cos. 


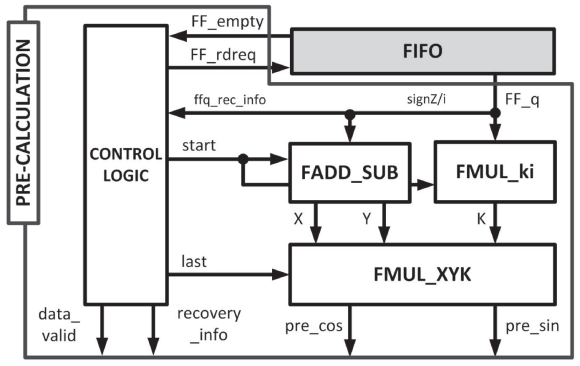

(a)

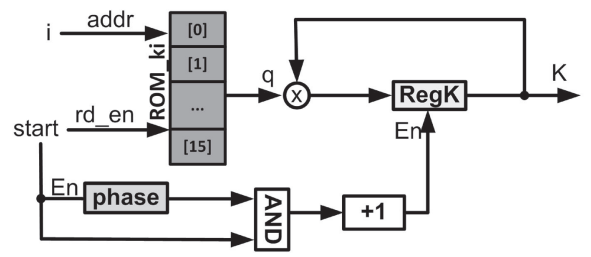

(c)

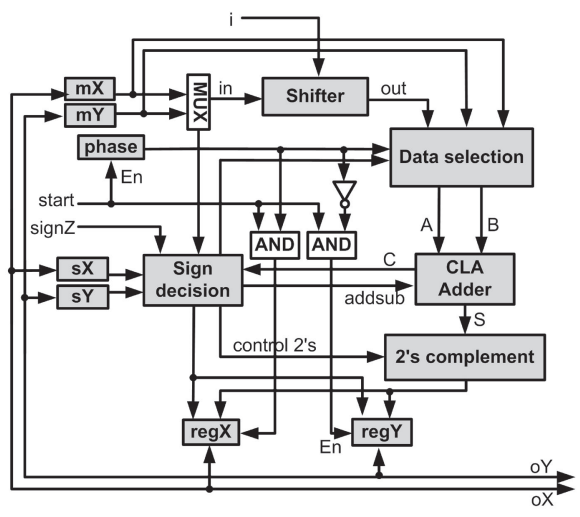

(b)

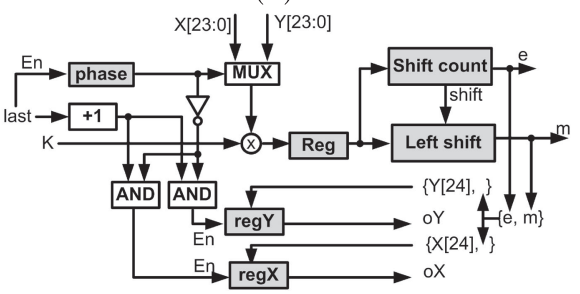

(d)

Fig. 7. The hardware architecture of (a) PREC circuit. (b) FADD_SUB circuit. (c) FMUL_ki circuit. (d) FMUL_XYK circuit.

\section{Experimental results}

The performance of the proposed CORDIC is evaluated in two aspects: algorithm and hardware design. The first assessment proves that proposed algorithm requires fewer iterations but achieves higher precision than the original CORDIC. The second assessment compares this work with the other floating-point systems in terms of latency and resource utilization.

\subsection{Evaluation of algorithm}

In order to assess the algorithm, 9001 angles, from $\left[-45^{\circ}, 45^{\circ}\right]$ are generated. The total number of iterations are observed at three different number of angle constants - namely $N$. As can be seen in Fig. 8, the proposed HA-CORDIC only requires maximum 4,6 , and 8 micro-rotations in case $N=8,12,16$, respectively. In other words, in the worst case, the HA-CORDIC is still $2.7 X, 2.4 X$, and $2.3 X$ faster than the original one. Moreover, on average, the latency improvements are $3.8 X, 3.5 X$, and $3.4 X$ at $N=8,12,16$, respectively.

The precision of two algorithms is measured by mean square error (MSE) of the residual angle. In fact, the more the residual angle is close to zero, the more the precision of sine/cosine can be achieve. It can be seen in Fig. 9, with the variation of $\mathrm{N}$ from 8 to 16 , the precision of both methods increases. Nevertheless, the proposed CORDIC always delivers smaller MSE value, 2.51e-7, than that from conventional one, 1.02e-6.

\subsection{Evaluation of hardware design}

The proposed HA-CORDIC is synthesized by Altera Quartus 14.0 with Stratix IV FPGA target. The latency, resource utilization (LUT, Register, Memory) and 

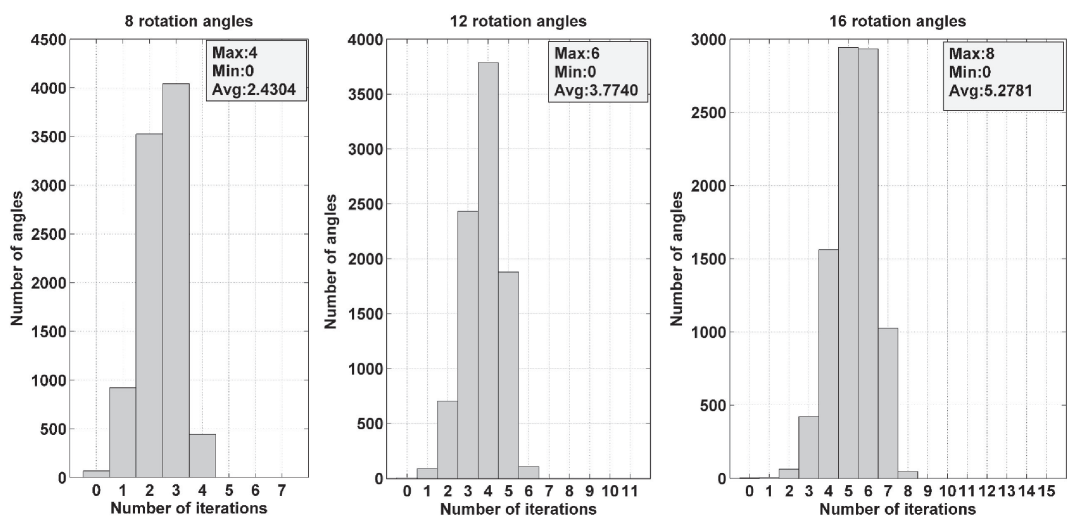

Fig. 8. The comparison in number of iterations in case of 8,12 , and 16 predetermined angle constants.

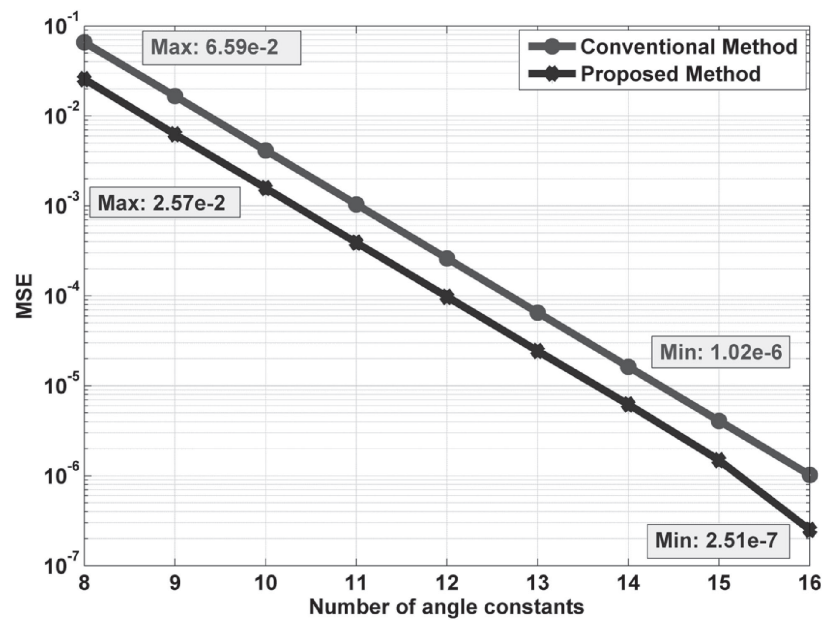

Fig. 9. The comparison in mean square error of residual angles.

Table II. The comparison between HA-CORDIC with other floatingpoint systems in terms of device family (A), latency (clocks) (B), frequency $(\mathrm{MHz})(\mathrm{C})$, lookup table $(\mathrm{D})$, register $(\mathrm{E})$, memory (F), and DSP (G).

\begin{tabular}{|c|c|c|c|c|c|c|c|}
\hline & [7] & {$[8]$} & [9] & [10] & {$[16]$} & [17] & This work \\
\hline \multirow{3}{*}{ (A) } & Xilinx & Xilinx & Xilinx & Altera & Xilinx & Altera & Altera \\
\hline & Virtex & Virtex & Virtex & Stratix & Virtex & Stratix & Stratix \\
\hline & 5 & 5 & 7 & II & 6 & IV & IV \\
\hline (B) & 93 & - & 130 & - & - & 36 & $12 / 20 / 26$ \\
\hline (C) & 86.1 & 133.8 & 280.0 & 195.1 & 253.5 & 258.3 & 175.7 \\
\hline (D) & 3,152 & 26,811 & 6,514 & 6,469 & 13,744 & 5,612 & 1,139 \\
\hline (E) & - & 16,274 & 4,725 & 5,372 & - & 4,231 & 498 \\
\hline (F) & 2,832 & - & 4,894 & - & - & 3,575 & 11 \\
\hline (G) & 0 & 2 & 9 & - & 96 & 32 & 8 \\
\hline
\end{tabular}


much shorter than the other results. At $N=16$, it costs 12, 20, and 26 clock cycles in best case (zero or one rotation), typical case (five rotations), and worst case (eight rotations). Besides, HA-CORDIC resource utilization is much better than the others regarding the operating frequency of $175.7 \mathrm{MHz}$.

\section{Conclusion}

In this paper, a low-latency hybrid adaptive CORDIC with floating-point precision is proposed. Because of adaptive angle selection, proposed HA-CORDIC needs fewer latency while achieves more precise trigonometric results rather than the original one. Furthermore, hybrid architecture, which includes a fixed-point input angle in degree and two floating-point sine/cosine outputs not only reduces the hardware resource in total but also enhances the results precision in general. The experiments show that the design is fully operational at $175.7 \mathrm{MHz}$ and costs 12 and 26 latency cycles in best and worst case, respectively, in case of $N=16$. Besides, HA-CORDIC is likely to integrate into other advanced systems easily due to given acknowledge signals and low resource consumption-1,139 LUTs and 489 registers only. 